\title{
Searching for Dark Matter in the Galactic Center with Gamma Rays
}

\author{
Simona Murgia* \\ University of California, Irvine \\ E-mail: smurgiaeuci.edu
}

The center of the Milky Way is predicted to be the brightest source of gamma rays produced by dark matter annihilation or decay. In recent years, claims have been made of an excess consistent with a dark matter annihilation signal in the data collected by the Fermi Large Area Telescope (Fermi-LAT) towards the Galactic center. Although these results are intriguing, the complexity involved in modeling the foreground and background emission from conventional astrophysical sources of gamma rays makes a conclusive interpretation of these results challenging. This is a summary of the current status of these searches, with some emphasis on the results from the Fermi-LAT Collaboration.

The 34th International Cosmic Ray Conference,

30 July- 6 August, 2015

The Hague, The Netherlands

${ }^{*}$ Speaker. 


\section{Introduction}

Evidence for dark matter (DM) is overwhelming [1]. From experimental data we can infer that DM constitutes most of the matter in the Universe and that it interacts very weakly, and at least gravitationally, with ordinary matter. Several extensions of the Standard Model of particle physics naturally predict the existence of new particles that are excellent DM candidates. A well motivated DM candidate is the WIMP (Weakly Interacting Massive Particle.) WIMPs couple with Standard Model particles with a strength of the order of the electroweak scale and, if produced in thermal equilibrium with Standard Model particles in the early Universe, will give rise to the correct DM relic density if their mass is of order $100 \mathrm{GeV} / c^{2}$ and they self-annihilate with a velocity averaged annihilation cross section of approximately $3 \times 10^{-26} \mathrm{~cm}^{3} / \mathrm{s}$. These values are only representative and WIMP models predict a broad range of masses and annihilation cross sections (see e.g. [1,2] for a review.) Because of its sensitivity and the energy range that it covers, Fermi-LAT [3, 4, 5], on board of the Fermi satellite, is a remarkable instrument to test WIMP scenarios where DM annihilates (or decays, with a lifetime of the order of the age of the Universe) producing $\gamma$-rays in the final state. The inner region of the Milky Way is predicted to be the brightest target for these searches. Cuspy DM profiles (Navarro, Frenk, and White (NFW) [6], Einasto [7]) in fact arise in cold DM-only simulation. However, a cored DM profile, which might occur when baryonic effects are included [8,9] or if DM is self-interacting [10], is not excluded by the data and the prospects for dark matter searches in this scenario would be significantly worse. In addition to the uncertainties in the distribution at the center of the DM halo, the inner Galaxy is very bright in gamma rays produced by known and more conventional astrophysical processes and the search for a DM signal is limited by the large uncertainties in modelling this fore-/background.

To escape the current limitations of the GC, much effort has been focused in searching for a signal of DM in the Milky Way dwarf spheroidal satellite galaxies (dSphs.) The DM content in dSphs dominates over ordinary matter by a factor that can be as large as $\sim 1000$ and it is inferred by the stellar kinematics, hence one does not rely as much on DM simulations to determine it. In addition, dSphs are not expected to host astrophysical sources of gamma rays other than potentially DM. dSphs are therefore considered to be one of the most promising targets for a DM signal in $\gamma$-rays. Notably, the non-detection to date of dSphs with the data collected by Fermi-LAT has significantly contributed to our understanding of DM by placing interesting constraints on its nature $[11,12]$. Recent constraints [13] reach and go beyond the thermal relic cross section of $3 \times 10^{-26} \mathrm{~cm}^{3} / \mathrm{s}$ for WIMPs with masses below $\sim 100 \mathrm{GeV} / c^{2}$ for annihilation into bottom quarks. The GC however is predicted to outshine dSphs by one order of magnitude or more (depending on the DM profile and search region), and a potential discovery could be currently concealed by the uncertainties in modeling the astrophysical fore-/background.

\section{Status of Dark Matter Searches in the Galactic Center with Fermi-LAT}

An excess in the Fermi-LAT data in the direction of the GC was first claimed in [14]. More recent analyses confirm the presence of the excess [15, 16, 17, 18, 19, 20, 21, 22]. The excess is claimed to be spatially consistent with DM annihilation for an NFW profile with slope $\gamma=1.1-1.3$ centered at the location of the GC supermassive black hole, Sgr A*. Deviations from a spheri- 
cally symmetric morphology are disfavored and the excess extends to at least $10^{\circ}$ from the Galactic plane. The spectrum is consistent with a WIMP mass of $\sim 50(\sim 10) \mathrm{GeV} / \mathrm{c}^{2}$ if annihilating predominantly into hadronic (leptonic) final states, with an annihilation cross-section compatible with a thermal relic. Alternative interpretations for this excess have been proposed. Notably, a population of unresolved pulsars is found plausible and compatible with the observed excess (see e.g. $[20,23,24]$.) More recently, this hypothesis has been tested with non-poissonian photon statistics template analysis [25, 26] and with wavelet decomposition [27]. These are promising ways to disentangle an extended component in the data that is more likely to originate from a population of discrete $\gamma$-ray emitters rather than with the continuous emission predicted for DM annihilation (although DM substructures would contribute to the discrete emission.) Finally, cosmic-ray (CR) proton or electron outbursts (e.g. [28, 29, 30]) interpretations have also been proposed, although they arguably require a larger degree of fine tuning to explain all aspects of the excess.

\section{Modeling of the Galactic Interstellar Emission and Results}

Regardless of the particular interpretation, modeling of the Galactic interstellar emission (IE) and point sources is crucial in the characterization of the excess emission. Limitations and uncertainties in modeling these emissions must be addressed to confirm the presence and properties of additional components in the data, dark matter or otherwise. The Galactic IE is generated by CR particles interacting with the interstellar gas and radiation field. The physical processes that contribute to this emission are: $\pi^{0}$-decay, i.e. inelastic scattering of CR nuclei with the interstellar gas producing $\pi^{0}$ 's and, in turn, $\gamma$-rays from their decay; bremsstrahlung by electrons and positrons on the interstellar gas; Inverse Compton (IC) scattering, i.e. CR electrons and positrons up-scattering the photons in the interstellar radiation field (ISRF) of the Galaxy to $\gamma$-ray energies. Modeling this emission requires knowledge of the origin and propagation of CRs, which is particularly uncertain for the GC region, where CR intensities, density of the ISRF and gas are highest and most uncertain and where there is a significant fore-/background contribution with long integration path over the entire Galactic disc. In addition, the many energetic sources near to or in the line of sight of the GC are difficult to disentangle from the IE. Two approaches have been most often employed to model the Galactic IE in the analysis of the Fermi-LAT GC data. One is to adopt the IE models (IEMs) provided by the Fermi-LAT Collaboration [31]. They are designed to flatten residuals over extended regions of the sky, for the study of point sources and sources with small extension in the Fermi-LAT data. These models often include patches to absorb positive residuals and since some of the patches are in and about the GC, the interpretation of additional extended excesses in this region is uncertain. The other approach is to use the CR propagation code GALPROP [32], which provides physically motivated models for the IE, but does not fully capture the complexity of the Galaxy. The spectrum of the GC excess is shown in Figure 1 for [21] (left), where the IEM from [31] is employed, and [22], where the IEM is modeled with GALPROP. In both cases the spectrum of the GC excess peaks at a few $\mathrm{GeV}$, however there is a strong dependence on the IEM at higher energies. Both of these analyses use established gamma-ray source catalogs to model point sources.

An alternative and novel approach to determine the IEM and point sources in the inner Galaxy has been employed by the Fermi-LAT collaboration [33]. Specialized IEMs are constructed for 

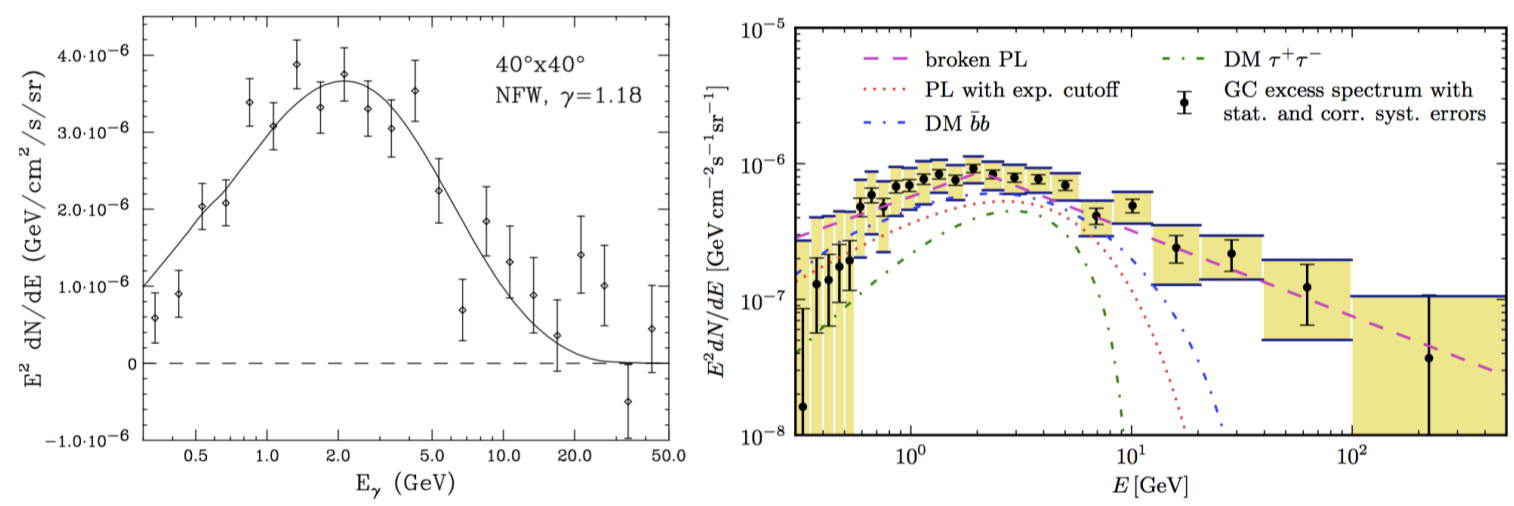

Figure 1: Spectrum of the Galactic center excess from [21] (left), and [22] (right).

a $15^{\circ} \times 15^{\circ}$ region about the direction of the GC to separate the emission from the inner $\sim 1 \mathrm{kpc}$ of the GC from the rest of the Galaxy in the $1-100 \mathrm{GeV}$ energy range. A catalog of point sources for the $15^{\circ} \times 15^{\circ}$ region is self-consistently constructed using these IEMs. This work and some of the related results are summarized below. The starting point to model the IE for the FermiLAT Collaboration GC analysis are IEMs from [34]. A major uncertainty affecting predictions of the IE toward the inner Galaxy is the spatial distribution of CR sources. In this work, the [35] pulsar distribution ("Pulsars") and the distribution of OB-stars ("OBstars" [36]) are used as proxies for the CR source distribution as they cover a broad range in its radial extent. Two IEMs from [34], corresponding to these two assumptions for the CR source distribution, are employed as baselines in this work and are further fit to the Fermi-LAT data (excluding the $15^{\circ} \times 15^{\circ}$ region about the GC) for improved fore-/background determination. The intensity maps, calculated by GALPROP in Galactocentric annuli, are used as templates for the IEM fitting procedure together with an isotropic component and a model for $\gamma$-ray emission associated with Loop I [37]. Two IEMs for each of the Pulsars and OBstars models - four in total - are constructed. The two variants for each model are termed "intensity-scaled"and "index-scaled". The normalization parameters for the templates are determined in a series of fits to the data, starting at high latitudes for the local components and then working from the outer Galaxy to the inner Galaxy, always fixing the already determined normalization parameters in subsequent fits. For the intensity-scaled variants only the normalizations of the individual intensity maps are allowed to change. For the index-scaled variants the same fitting procedure is followed, but additional degrees of freedom are allowed to the spectrum of the gas-related IE when fitting to the annuli interior to the solar circle. This is motivated by the fact that the residuals indicate that the spectrum of the IEM related to the CR nuclei/gas interaction ( $\pi^{0}$-decay) in this region may be too soft. After this scaling, the agreement between data and model has improved for all baseline models and scaling procedure. It is not straightforward to identify a best IEM and consequently, all 4 (Pulsars/OBstars, intensity-/indexscaled) IEMs are used to estimate the fore-/background toward the $15^{\circ} \times 15^{\circ}$ region about the GC.

The wavelet based algorithm PGWave [38], which makes minimal assumption on the background, is employed to initially extract the position of point source candidates in the $15^{\circ} \times 15^{\circ}$ 
region, while their spectral properties and refinements on their position are determined by a maximum likelihood fit for each of the fore-/background models and concurrently with the IE in the $15^{\circ} \times 15^{\circ}$ region. The intensities for the IE from the innermost $1 \mathrm{kpc}$ are determined by fitting the data in this region concurrently with the point source candidates, while the fore-/background models are held constant. This procedure is repeated until no significant point-like excesses remain in the residuals. Figures 2 and 3 show the differential flux and residuals for the Pulsars IEMs (the OBstars IEMs display very similar trends.)
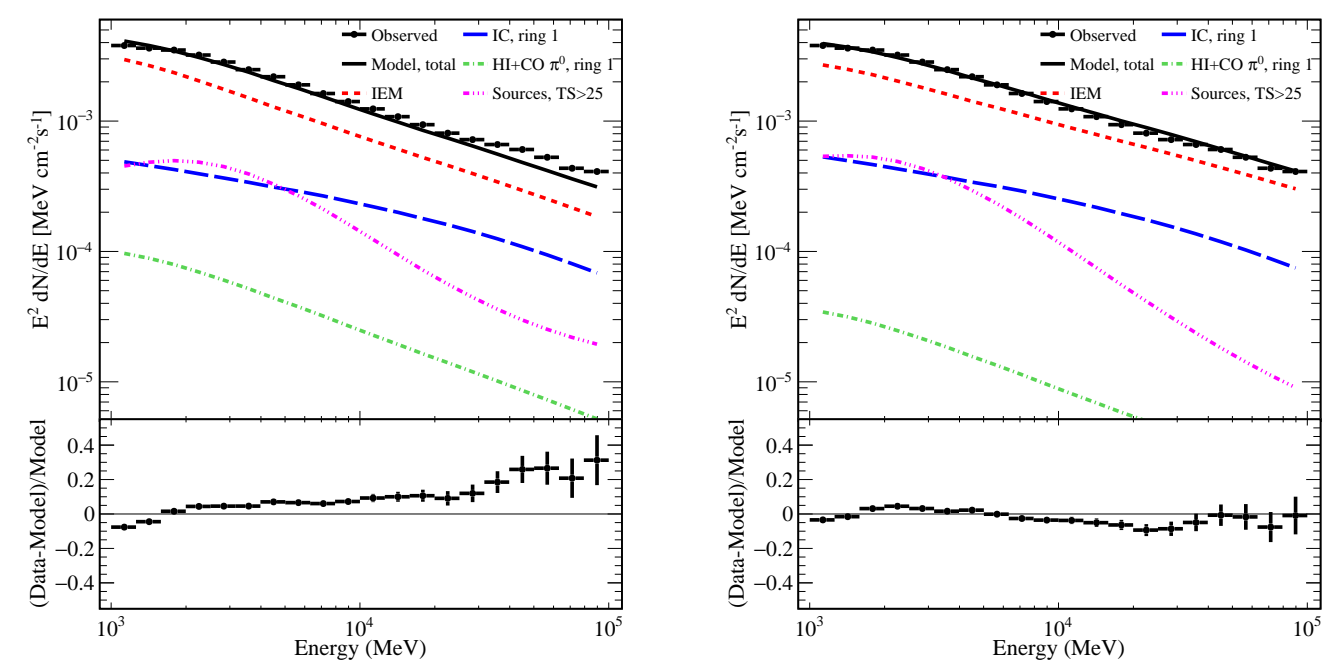

Figure 2: Differential fluxes for the $15^{\circ} \times 15^{\circ}$ region about the GC for the Pulsars intensity- (left) and indexscaled (right) IEMs. Line styles: solid (total model), long-dash (IC, annulus 1), dot-dash (H I and CO gas $\pi^{0}$-decay, annulus 1), dot-dot-dot-dash (point sources), dash (Galactic IE excluding annulus 1 for IC, H I and $\mathrm{CO}$ gas $\pi^{0}$-decay). Solid circles: data.

The fore-/background are found to account for most of the emission in the region. The fitted IC for the inner $1 \mathrm{kpc}$ is bright, more than predicted by the baseline models (6-30x), which might imply more intense ISRF and/or CR electron density over the inner region. The gas $\pi^{0}$ intensities however are subdominant and much dimmer than predicted. For the intensity-scaled variant of the models, the data-model agreement is within $5-10 \%$ over the $15^{\circ} \times 15^{\circ}$ region up to $10 \mathrm{GeV}$. The models are too bright below $2 \mathrm{GeV}$ and this disagreement is correlated with the Galactic plane, which might be pointing to an issue with the modeling of the spectra of the CR nuclei. The agreement is generally better for the index-scaled models. Above $\sim 10 \mathrm{GeV}$, the intensity-scaled models increasingly underpredict the data, while the index-scaled models overpredict the data. At a few $\mathrm{GeV}$ all models underpredict the data and the excess appears to be extended and distributed around the GC, which might indicate the presence of a new extended component in the data. The possibility that an additional component centered at the GC contributes to the data is therefore explored. Because the excess emission in the few $\mathrm{GeV}$ range is distributed around the GC, templates that peak there are considered: a set of two-dimensional Gaussians with varying HWHM; spatial templates to model the predicted distribution for $\gamma$-rays produced by DM annihilation or decay following a NFW profile (the square of the NFW profile is used as a template for DM annihilation); unresolved 

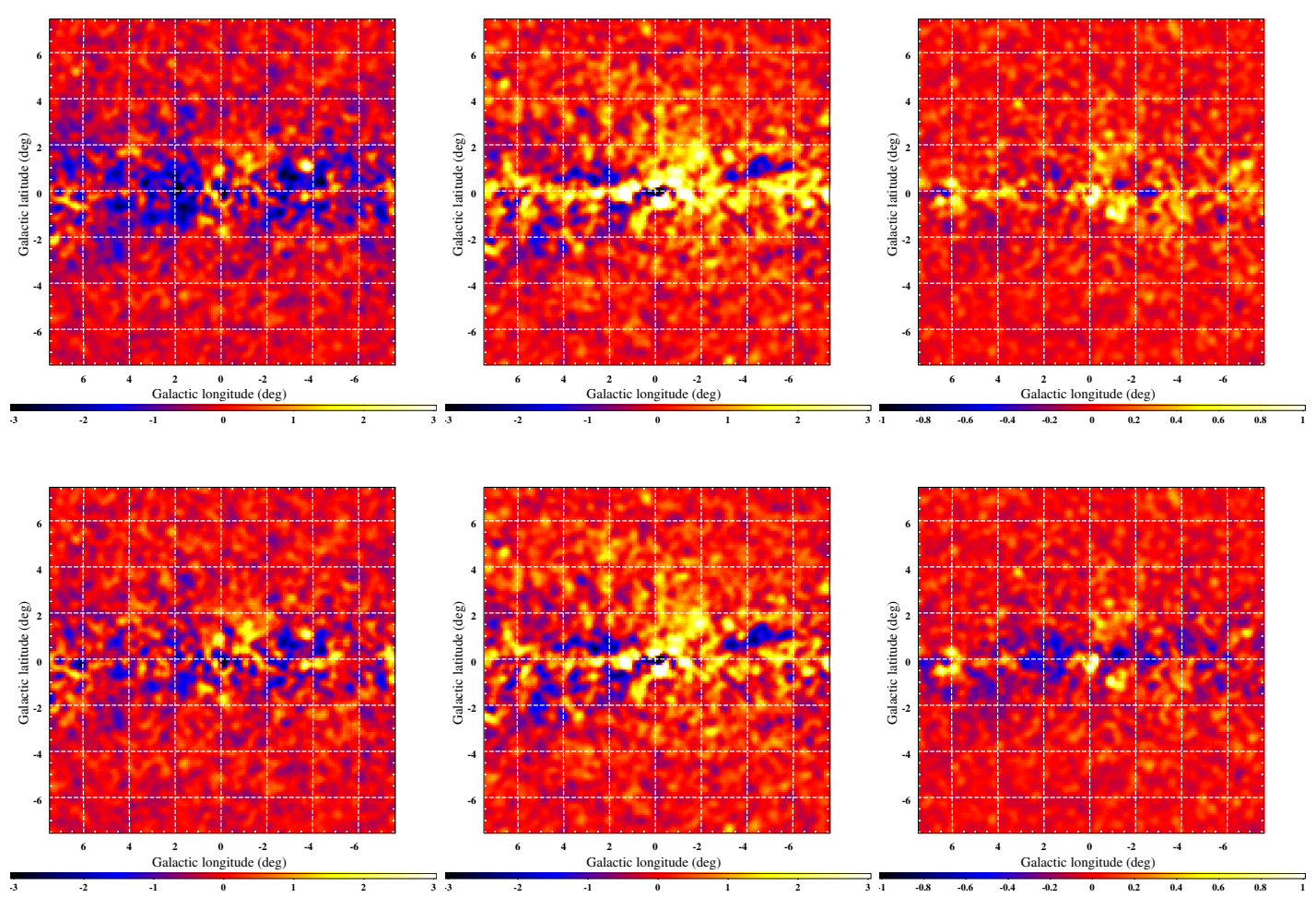

Figure 3: Residual counts for the $15^{\circ} \times 15^{\circ}$ region about the GC for the Pulsars IEMs for energy ranges $1-1.6 \mathrm{GeV}$ (left), $1.6-10 \mathrm{GeV}$ (middle), and >10 GeV (right) for the intensity- (top) and index-scaled (bottom) variants. The colour scale is in counts/ $0.1 \mathrm{deg}^{2}$ pixel.

population of $\gamma$-ray point sources such as pulsars distributed along the Galactic plane. For each of the spatial templates listed above, the spectrum is modelled with an exponential cut-off power law. This form has some flexibility to model a pulsar or a DM annihilation spectrum without supposing specific scenarios. Also, more degrees of freedom are allowed to model the spectrum by employing a power-law function per energy bin, with 10 bins equally spaced in logarithmic energy over the $1-100 \mathrm{GeV}$ energy range. For each of the spatial templates listed above and for each of the IEMs, a maximum-likelihood fit is made in the $15^{\circ} \times 15^{\circ}$ region. Among all the templates that have been tested, the DM annihilation template yields the most significant improvements in the data-model agreement for all fore-/background IEMs. Figures 4 and 5 show the differential flux and residuals for the Pulsars index-scaled IEM, which yields the best agreement over the full energy range. A broad range for the best-fit parameters of the spectral model is found depending on the choice of IEM. This can be seen in Fig. 6, which shows the flux spectral envelopes for all IEMs. These cover a broader range compared with earlier determinations of the GC excess spectrum. The variation is not easily ascribed to a covariance with only a single component of the model that is fitted over the $15^{\circ} \times 15^{\circ}$ region. For example, the annulus $1 \mathrm{IC}$ and $\mathrm{H}$ I-related $\pi^{0}$-decay normalizations adjust in the fit to compensate for the additional template. The interplay between the centrally peaked positive residual template and the IE components is not surprising. Because the IC component is maximally peaked toward the GC for all IEMs an additional template that is also peaked there will also be attributed some flux when fit. But the spectral parameters of the residual template are not 

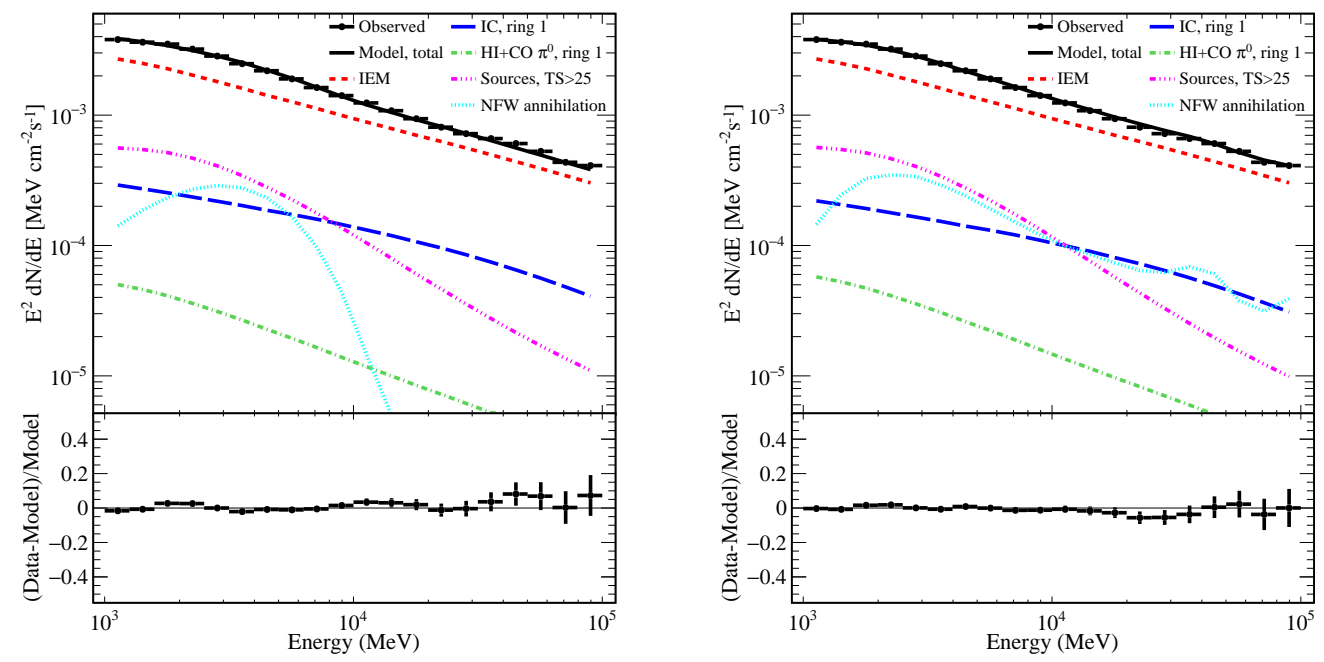

Figure 4: Differential fluxes for the $15^{\circ} \times 15^{\circ}$ region about the GC for the Pulsars index-scaled IEM using an DM annihilation distribution centered on the GC as an additional spatial template for the maximumlikelihood fit with spectrum modeled with a exponential cut-off power law function (left) or a power-law per energy bin (right). Line styles: solid (total model), long-dash (IC, annulus 1), dot-dash (H I and CO gas $\pi^{0}$-decay, annulus 1), dot-dot-dot-dash (point sources), dash (Galactic IE excluding annulus 1 for IC, H I and CO gas $\pi^{0}$-decay), dot (DM annihilation). Solid circles: data.
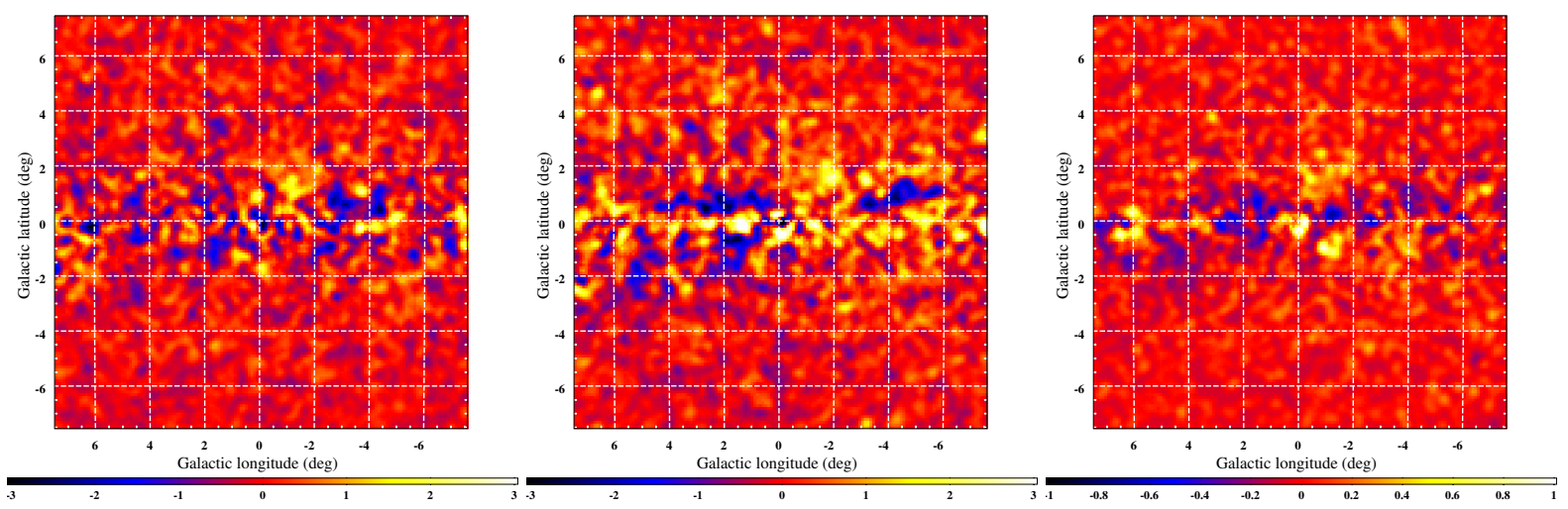

Figure 5: Residual counts for the $15^{\circ} \times 15^{\circ}$ region about the GC for the Pulsars index-scaled IEM together with the DM annihilation template for energy ranges $1-1.6 \mathrm{GeV}$ (left), $1.6-10 \mathrm{GeV}$ (middle), and > $10 \mathrm{GeV}$ (right). The colour scale is in counts $/ 0.1 \mathrm{deg}^{2}$ pixel.

solely determined by the fit with the IE components and point sources over the inner region about the GC; the fore-/background IE has an effect as well. The prescriptive method of determining the fore-/background IE, together with the self-consistent treatment of the point sourcess ${ }^{1}$ and IE for the inner $\sim 1 \mathrm{kpc}$ about the GC allows the least biased estimate to-date to be made of the positive residual emission about the GC.

\footnotetext{
${ }^{1}$ Note: this includes the sub-threshold point source candidates and those that satisfy the $T S>25$ criterion for "detection" used for the 1FIG.
} 

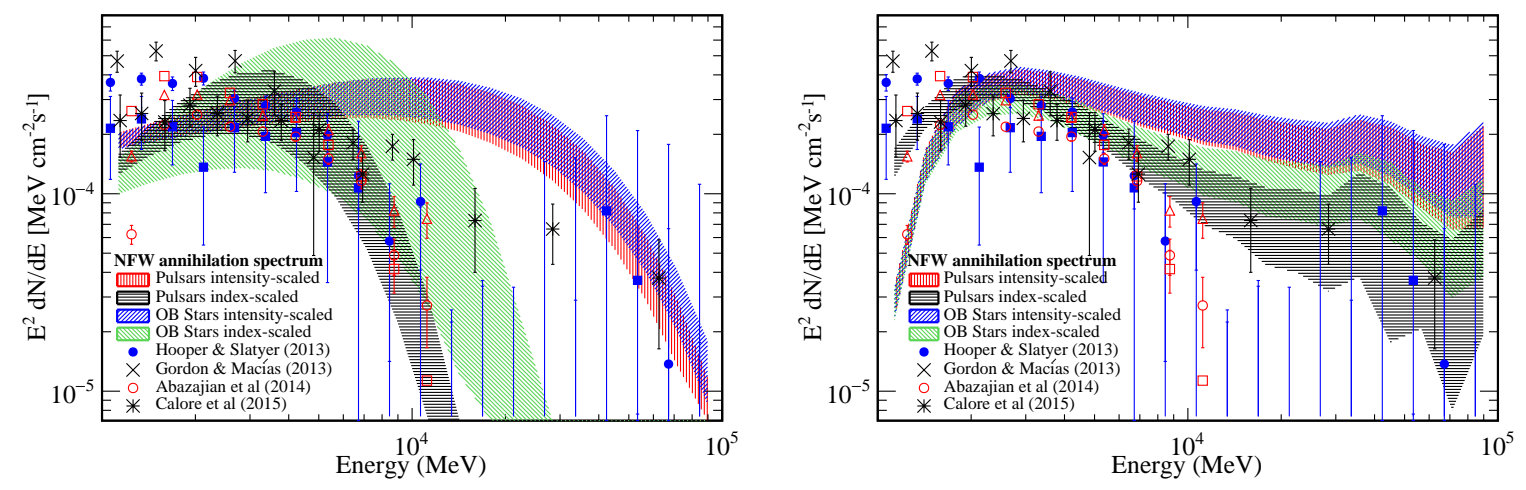

Figure 6: Differential fluxes for the $15^{\circ} \times 15^{\circ}$ region about the GC of the NFW component with spectrum modelled with an exponential cut-off power law (left) or with a power-law per energy band (right). The envelopes include the fit uncertainties for the normalization and spectral index. Hatch styles: Pulsars, intensity-scaled (red, vertical); Pulsars, index-scaled (black, horizontal); OBstars, intensity-scaled (blue, diagonal-right); OBstars, index-scaled (green, diagonal-left). Results from selected other works are overlaid. Filled symbols: [18], different symbols bracket the results obtained when different regions of the sky are considered in the fit; Angled crosses: [19]; Open symbols: [20], front-converting events shown with triangles, front- and back-converting events shown with squares and circles, depending on the modelling of the fore-/background. Stars : [22]. Note: the overlaid results are rescaled to the DM content over the $15^{\circ} \times 15^{\circ}$ region for an NFW profile with index $\gamma=1$.

Although a large formal statistical significance may be indicated for the detection of a DM component, note that fitting a centrally peaked profile does not account for all of the excess emission over the $15^{\circ} \times 15^{\circ}$ region. Ascribing a singular origin to such a residual component is premature given the limited constraints on the other emission components over the $15^{\circ} \times 15^{\circ}$ region. This can be seen in Fig. 5, which shows the residual counts for the NFW template and IEM with the best spectral residuals (Pulsars index-scaled). Qualitatively, the remainder does not appear distributed symmetrically about the GC below $10 \mathrm{GeV}$, and still has extended positive residuals even at higher energies along and about the plane. A complete assessment of the uncertainties (see [33] for a discussion) is required to understand the nature of its spatial and spectral parameters. The current work demonstrates that even in the optimistic scenario where the presence of a DM component in the data might be established based on the spatial distribution of the associated $\gamma$-ray emission, important information on the DM particle such as its mass and annihilation spectrum is strongly dependent on the IEM. This was first demonstrated by [39] using preliminary results based on this work.

\section{References}

[1] G. Bertone, D. Hooper and J. Silk, "Particle dark matter: Evidence, candidates and constraints," Phys. Rept. 405, 279 (2005) [hep-ph/0404175].

[2] J. L. Feng, "Dark Matter Candidates from Particle Physics and Methods of Detection," Ann. Rev. Astron. Astrophys. 48, 495 (2010) [arXiv:1003.0904 [astro-ph.CO]]. 
[3] W. B. Atwood et al. [LAT Collaboration], "The Large Area Telescope on the Fermi Gamma-ray Space Telescope Mission,” Astrophys. J. 697, 1071 (2009) [arXiv:0902.1089 [astro-ph.IM]].

[4] A. A. Abdo et al. [Fermi-LAT Collaboration], "The On-orbit calibrations for the Fermi Large Area Telescope,” Astropart. Phys. 32, 193 (2009) [arXiv:0904.2226 [astro-ph.IM]].

[5] M. Ackermann et al. [Fermi-LAT Collaboration], "The Fermi Large Area Telescope On Orbit: Event Classification, Instrument Response Functions, and Calibration,” Astrophys. J. Suppl. 203, 4 (2012) [arXiv:1206.1896 [astro-ph.IM]].

[6] J. F. Navarro, C. S. Frenk and S. D. M. White, “The Structure of cold dark matter halos," Astrophys. J. 462, 563 (1996) [astro-ph/9508025].

[7] V. Springel, J. Wang, M. Vogelsberger, A. Ludlow, A. Jenkins, A. Helmi, J. F. Navarro and C. S. Frenk et al., "The Aquarius Project: the subhalos of galactic halos," Mon. Not. Roy. Astron. Soc. 391, 1685 (2008) [arXiv:0809.0898 [astro-ph]].

[8] F. Governato, A. Zolotov, A. Pontzen, C. Christensen, S. H. Oh, A. M. Brooks, T. Quinn and S. Shen et al., "Cuspy No More: How Outflows Affect the Central Dark Matter and Baryon Distribution in Lambda CDM Galaxies,” Mon. Not. Roy. Astron. Soc. 422, 1231 (2012) [arXiv:1202.0554 [astro-ph.CO]].

[9] A. V. Maccio', G. Stinson, C. B. Brook, J. Wadsley, H. M. P. Couchman, S. Shen, B. K. Gibson and T. Quinn, "Halo expansion in cosmological hydro simulations: towards a baryonic solution of the cusp/core problem in massive spirals,” [arXiv:1111.5620 [astro-ph.CO]].

[10] M. Rocha, A. H. G. Peter, J. S. Bullock, M. Kaplinghat, S. Garrison-Kimmel, J. Onorbe and L. A. Moustakas, "Cosmological Simulations with Self-Interacting Dark Matter I: Constant Density Cores and Substructure,” Mon. Not. Roy. Astron. Soc. 430, 81 (2013) [arXiv:1208.3025 [astro-ph.CO]].

[11] M. Ackermann et al. [Fermi-LAT Collaboration], "Constraining Dark Matter Models from a Combined Analysis of Milky Way Satellites with the Fermi Large Area Telescope,” Phys. Rev. Lett. 107, 241302 (2011) [arXiv:1108.3546 [astro-ph.HE]].

[12] A. Geringer-Sameth and S. M. Koushiappas, "Exclusion of canonical WIMPs by the joint analysis of Milky Way dwarfs with Fermi,” Phys. Rev. Lett. 107, 241303 (2011) [arXiv:1108.2914 [astro-ph.CO]].

[13] M. Ackermann et al. [Fermi-LAT Collaboration], "Searching for Dark Matter Annihilation from Milky Way Dwarf Spheroidal Galaxies with Six Years of Fermi-LAT Data,” arXiv:1503.02641 [astro-ph.HE].

[14] L. Goodenough and D. Hooper, "Possible Evidence For Dark Matter Annihilation In The Inner Milky Way From The Fermi Gamma Ray Space Telescope," arXiv:0910.2998 [hep-ph].

[15] D. Hooper and L. Goodenough, "Dark Matter Annihilation in The Galactic Center As Seen by the Fermi Gamma Ray Space Telescope,” Phys. Lett. B 697, 412 (2011) [arXiv:1010.2752 [hep-ph]].

[16] D. Hooper and T. Linden, “On The Origin Of The Gamma Rays From The Galactic Center," Phys. Rev. D 84, 123005 (2011) [arXiv:1110.0006 [astro-ph.HE]].

[17] K. N. Abazajian and M. Kaplinghat, "Detection of a Gamma-Ray Source in the Galactic Center Consistent with Extended Emission from Dark Matter Annihilation and Concentrated Astrophysical Emission," Phys. Rev. D 86, 083511 (2012) [arXiv:1207.6047 [astro-ph.HE]]. 
[18] D. Hooper and T. R. Slatyer, "Two Emission Mechanisms in the Fermi Bubbles: A Possible Signal of Annihilating Dark Matter," [arXiv:1302.6589 [astro-ph.HE]].

[19] C. Gordon and O. Macias, "Dark Matter and Pulsar Model Constraints from Galactic Center Fermi-LAT Gamma Ray Observations,” [arXiv:1306.5725 [astro-ph.HE]].

[20] K. N. Abazajian, N. Canac, S. Horiuchi and M. Kaplinghat, "Astrophysical and Dark Matter Interpretations of Extended Gamma-Ray Emission from the Galactic Center," arXiv:1402.4090 [astro-ph.HE].

[21] T. Daylan, D. P. Finkbeiner, D. Hooper, T. Linden, S. K. N. Portillo, N. L. Rodd and T. R. Slatyer, "The Characterization of the Gamma-Ray Signal from the Central Milky Way: A Compelling Case for Annihilating Dark Matter," arXiv:1402.6703 [astro-ph.HE].

[22] F. Calore, I. Cholis and C. Weniger, "Background model systematics for the Fermi GeV excess," arXiv:1409.0042 [astro-ph.CO].

[23] R. M. O’Leary, M. D. Kistler, M. Kerr and J. Dexter, "Young Pulsars and the Galactic Center GeV Gamma-ray Excess,” arXiv:1504.02477 [astro-ph.HE].

[24] T. D. Brandt and B. Kocsis, "Disrupted Globular Clusters Can Explain the Galactic Center Gamma Ray Excess," arXiv:1507.05616 [astro-ph.HE].

[25] S. K. Lee, M. Lisanti and B. R. Safdi, "Distinguishing Dark Matter from Unresolved Point Sources in the Inner Galaxy with Photon Statistics,” JCAP 1505, no. 05, 056 (2015) [arXiv:1412.6099 [astro-ph.CO]].

[26] S. K. Lee, M. Lisanti, B. R. Safdi, T. R. Slatyer and W. Xue, "Evidence for Unresolved Gamma-Ray Point Sources in the Inner Galaxy,” arXiv:1506.05124 [astro-ph.HE].

[27] R. Bartels, S. Krishnamurthy and C. Weniger, "Strong support for the millisecond pulsar origin of the Galactic center GeV excess," arXiv:1506.05104 [astro-ph.HE].

[28] E. Carlson and S. Profumo, "Cosmic Ray Protons in the Inner Galaxy and the Galactic Center Gamma-Ray Excess,” Phys. Rev. D 90, no. 2, 023015 (2014) [arXiv:1405.7685 [astro-ph.HE]].

[29] J. Petrovic, P. D. Serpico and G. Zaharijas, "Galactic Center gamma-ray "excess" from an active past of the Galactic Centre?,' JCAP 1410, no. 10, 052 (2014) [arXiv:1405.7928 [astro-ph.HE]].

[30] I. Cholis, C. Evoli, F. Calore, T. Linden, C. Weniger and D. Hooper, "The Galactic Center GeV Excess from a Series of Leptonic Cosmic-Ray Outbursts," arXiv:1506.05119 [astro-ph.HE].

[31] http://fermi.gsfc.nasa.gov/ssc/data/access/lat/BackgroundModels.html

[32] http://galprop.stanford.edu

[33] M. Ajello et al. [Fermi-LAT Collaboration], "Fermi-LAT Observations of High-Energy Gamma-Ray Emission Toward the Galactic Center,” arXiv:1511.02938 [astro-ph.HE].

[34] M. Ackermann et al. [Fermi-LAT Collaboration], "Fermi-LAT Observations of the Diffuse Gamma-Ray Emission: Implications for Cosmic Rays and the Interstellar Medium,” Astrophys. J. 750 (2010) 1044 [arXiv:1202.4039 [astro.ph]]

[35] I. Yusifov and I. Kucuk, "Revisiting the radial distribution of pulsars in the galaxy," Astron. Astrophys. 422, 545 (2004) doi:10.1051/0004-6361:20040152 [astro-ph/0405559].

[36] L. Bronfman, S. Casassus, J. May and L. A. Nyman, “The radial distribution of ob star formation in the galaxy," Astron. Astrophys. 358, 521 (2000) [astro-ph/0006104]. 
[37] M. Wolleben, “A New Model For The Loop-I (The North Polar Spur) Region,” Astrophys. J. 664, 349 (2007) doi:10.1086/518711 [arXiv:0704.0276 [astro-ph]].

[38] F. Damiani, A. Maggio, G. Micela, and S. Sciortino. "A Method Based on Wavelet Transforms for Source Detection in Photon-counting Detector Images. I. Theory and General Properties,” Astrophys. J. 483 (1997) 350

[39] P. Agrawal, B. Batell, P. J. Fox and R. Harnik, "WIMPs at the Galactic Center," JCAP 1505, 011 (2015) doi:10.1088/1475-7516/2015/05/011 [arXiv:1411.2592 [hep-ph]]. 